\title{
The Dirac operator on Lorentzian spin manifolds and the Huygens property
}

\section{H. Baum}

Sfb 288 Preprint No. 214

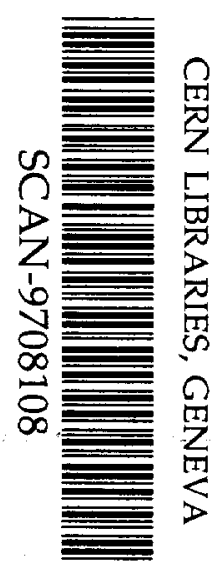

$$
\operatorname{sing} 735
$$

Diese Arbeit ist mit Unterstützung des von der Deutschen Forschungsgemeinschaft getragenen Sonderforschungsbereiches 288 entstanden und als Manuskript vervielfältigt worden.

Berlin, August 1996

The list of preprints of the Sonderforschungsbereich 288 is available at:

http://www-sfb288. math.tu-berlin.de 


\title{
The Dirac operator on Lorentzian spin manifolds and the Huygens property
}

\author{
Helga Baum
}

August 9, 1996

\begin{abstract}
We consider the Dirac operator $D$ of a Lorentzian spin manifold of even dimension $n \geq 4$. We prove, that the square $D^{2}$ of the Dirac operator on plane wave manifolds and the shifted operator $D^{2}-K$ on Lorentzian space forms of constant sectional curvature $K$ are of Huygens type. Furthermore, we study the Huygens property for coupled Dirac operators on 4-dimensional Lorentzian spin manifolds.
\end{abstract}

\section{Introduction}

It is a familiar phenomenon that waves propagate quite different in 2 and 3 dimensions. When a pebble falls into water at a certain point $x_{0}$, circular waves around $x_{0}$ are formed. A given point near $x_{0}$ will be hit by an initial ripple and later by residual waves. 3-dimensionally, the situation is quite different. If we produce a sound localized at the neighbourhood of a point $x_{0}$ then someone near $x_{0}$ will hear the sound during a certain time interval but no longer. There are no residual waves like those present on the water surface.

The mathematical reason for this different behaviour is a special property of the fundamental solution of the wave operator $\square_{m}$ of the $\mathbb{R}^{m}$ in dimension $m=3$. Whereas in general the forward fundamental solution of $\square_{m}$ with respect to the point $o \in \mathbb{R}^{m+1}$ is supported in the future cone $\mathcal{J}_{+}(o)=\left\{(x, t) \in \mathbb{R}^{m} \times \mathbb{R} \mid\|x\| \leq t\right\}$ the forward fundamental solution in dimension $m=3$ and each other odd dimension $m \geq 3$ is supported even in the light cone $\mathcal{C}_{+}(o)=\left\{(x, t) \in \mathbb{R}^{m} \times \mathbb{R} \mid\|x\|=t\right\}$. This produces a "sharp" wave propagation. Operators describing a "sharp" wave propagation such as $\square_{3}$ are called operators of Huygens type or shortly Huygens operators. In 1923, in his Yale Lectures, J. Hadamard posed the problem of finding all normally hyperbolic operators of Huygens type (see [Had23], p.236). In spite of its age this problem is still far from being completely solved. For results of the last thirty years and methods developed to treat this problem see [Gün88, Gün91, Wün94, BV94, BK96].

In this paper we consider the Huygens property for the square of the Dirac operator of a Lorentzian spin manifold.

Dirac operators on 4-dimensional analytic Lorentzian spin manifolds were studied by V.Wünsch. He proved the following result: 
Theorem 1 ([Wün78], Cor.3.3, [Wün79], Prop.5.6, [Wün80], Prop.2.11)

Let us denote by $D: \Gamma(S) \longrightarrow \Gamma(S)$ the Dirac operator of a 4-dimensional analytic Lorentzian spin manifold $\left(M^{4}, g\right)$ and let $f$ be a smooth function on $M$. If the operator $D^{2}-f: \Gamma(S) \longrightarrow \Gamma(S)$ is of Huygens type, then the scalar curvature $R$ of $(M, g)$ is constant and equals $12 f$.

If $R$ is constant and non zero, then $D^{2}-\frac{1}{12} R$ is of Huygens type if and only if $\left(M^{4}, g\right)$ has constant sectional curvature.

If $R$ is identically zero, then $D^{2}$ is of Huygens type if and only if $\left(M^{4}, g\right)$ is conformally flat or a plane wave manifold.

According to this Theorem there are exactly 3 classes of 4-dimensional analytic Lorentzian spin manifolds on which $D^{2}$ (or a shift of it) is of Huygens type: the Lorentzian space forms, the plane wave manifolds and the conformally flat manifolds of vanishing scalar curvature.

In this paper we consider Lorentzian spin manifolds $\left(M^{n}, g\right)$ of even dimension $n \geq 4$. Let us denote by $D$ the Dirac operator of $\left(M^{n}, g\right)$. We prove, that the operator $D^{2}-K$ is of Huygens type if $\left(M^{n}, g\right)$ has constant sectional curvature $K$ (Theorem 7) and that $D^{2}$ is of Huygens type if $\left(M^{n}, g\right)$ is a plane wave manifold (Theorem 10 ). It would be interesting to know wether $D^{2}$ on conformally flat manifolds with vanishing scalar curvature in even dimension $n \geq 6$ is of Huygens type too and to find new classes of Lorentzian spin manifolds of even dimension $n \geq 6$ on which $D^{2}$ (or a shift of it) is of Huygens type.

Finally we consider the square of twisted Dirac operators on 4-dimensional manifolds. We prove that the Huygens property of these operators implies that the coupling connection is flat and that the manifold belongs to one of the 3 above mentioned classes of Lorentzian manifolds (Theorem 11).

In Chapter 2 we recall the definition of Huygens operators and describe conditions for normally hyperbolic operators to be of Huygens type. In Chapter 3 we discuss the Dirac operator on Lorentzian space forms and in Chapter 4 the Dirac operator on plane wave manifolds. Chapter 5 deals with coupled Dirac operators on 4-dimensional manifolds.

\section{Huygens operators}

We first recall the definition of normally hyperbolic operators and the properties of their fundamental solutions.

Definition 1 Let $M^{n}$ be a smooth n-dimensional manifold, $(E, p, M)$ a real or complex vector bundle over $M . A$ differential operator $P: \Gamma(E) \longrightarrow \Gamma(E)$ of second order on $E$ is called normally hyperbolic if there exists a Lorentzian metric such that the principal symbol $\sigma(P)$ of $P$ is given by $\sigma(P)_{x}(\xi)=-g_{x}(\xi, \xi) I d_{E_{x}}$, where $x \in M$ and $\xi \in T M^{*} \backslash 0$. 
Remark: In local coordinates on $M$ and a local trivialization of $E$ a normally hyperbolic operator can be expressed in the form

$$
P=-\sum_{i, j=1}^{n} g^{i j}(x) \frac{\partial^{2}}{\partial x_{i} \partial x_{j}}+\sum_{k=1}^{n} A^{k}(x) \frac{\partial}{\partial x_{k}}+B(x),
$$

where $\left(g^{i j}\right)$ is the inverse matrix of the coefficients of the Lorentzian metric.

Let $\left(M^{n}, g\right)$ be an n-dimensional oriented Lorentzian manifold and let us denote by $P$ the $S O(n, 1)$-principal bundle of all positive oriented orthonormal frames. A spinor structure of $\left(M^{n}, g\right)$ is a reduction $(Q, f)$ of $P$ with respect to the double covering of the special orthogonal group $S O(n, 1)$ by the spin group $S p i n(n, 1)$. A Lorentzian spin manifold is an oriented Lorentzian manifold with a fixed spinor structure $(Q, f)$. Let us denote by $\Delta_{n, 1}$ the spinor representation of $\operatorname{Spin}(n, 1)$. The complex vector bundle $S:=Q \times{ }_{S \sin (n, 1)} \Delta_{n, 1}$ associated to the principal bundle $Q$ is called spinor bundle of the spin manifold $\left(M^{n}, g\right)$. It is of complex dimension $N=2^{\left[\frac{n}{2}\right]}$. The Levi-Civita connection of $\left(M^{n}, g\right)$ defines a covariant derivative $\nabla^{S}$ in the spinor bundle $S$ given by the formula

$$
\nabla_{X}^{S} \varphi=X(\varphi)+\frac{1}{2} \sum_{k<l} \varepsilon_{k} \varepsilon_{l} \omega_{k l}(X) s_{k} \cdot s_{l} \cdot \varphi
$$

where $\left(s_{1}, \ldots, s_{n}\right)$ is a local orthonormal frame, $\varepsilon_{j}=g\left(s_{j}, s_{j}\right)= \pm 1, \omega_{k l}=g\left(\nabla s_{k}, s_{l}\right)$ are the connection forms of the Levi-Civita connection $\nabla$ with respect to $\left(s_{1}, \ldots, s_{n}\right)$ and - denotes the Clifford multiplication. Then the Dirac operator of the spin manifold $\left(M^{n}, g\right)$ is defined as the composition of the spinor derivative with the Clifford multiplication $\mu$

$$
D: \Gamma(S) \stackrel{\nabla^{S}}{\longrightarrow} \Gamma\left(T^{*} M \otimes S\right) \stackrel{g}{=} \Gamma(T M \otimes S) \stackrel{\mu}{\longrightarrow} \Gamma(S) .
$$

In local coordinates $D$ can be expressed by

$$
D \varphi=\sum_{i=1}^{n} \varepsilon_{i} s_{i} \cdot \nabla_{s_{i}}^{S} \varphi
$$

Using the spinor calculus it is easy to see, that the square $D^{2}$ of the Dirac operator is normally hyperbolic. (For details see [Bau81] or [LM89]).

Definition 2 An open subset $\Omega$ of $M^{n}$ is called a geodesically normal domain if it is a normal neighbourhood for each of its points. In particular, for any $x, y \in \Omega$ there exists a unique geodesic $c:[0,1] \longmapsto \Omega$ with $c(0)=x, c(1)=y$. Let $\sigma: \Omega \times \Omega \longrightarrow \mathbb{R}$ be the quadratic geodesic distance function defined by $\sigma(x, y):=g_{c(t)}\left(c^{\prime}(t), c^{\prime}(t)\right)$, where $c:[0,1] \rightarrow \Omega$ is the unique geodesic in $\Omega$ joining $x$ with $y$.

Let $\Omega \subset M^{n}$ be a time oriented geodesically normal domain and let $x_{0}$ be in $\Omega$. Then

$$
\begin{array}{ll}
\mathcal{J}_{+}^{\Omega}\left(x_{0}\right):=\{x \in \Omega \mid & \text { the unique geodesic in } \Omega \text { from } x_{0} \text { to } x \text { is causal } \\
& \text { and future oriented }\}
\end{array}
$$


is called the future of $x_{0} \in \Omega$ and

$$
\mathcal{C}_{+}^{\Omega}\left(x_{0}\right):=\partial \mathcal{J}_{+}^{\Omega}\left(x_{0}\right)=\left\{x \in \Omega \mid \text { the unique geodesic in } \Omega \text { from } x_{0} \text { to } x\right.
$$

is light like and future oriented $\}$

is called the future light cone of $x_{0} \in \Omega$. Similarly, we define the past $\mathcal{J}_{-}^{\Omega}\left(x_{0}\right)$ and the past light cone $\mathcal{C}_{-}^{\Omega}\left(x_{0}\right)$, where now the geodesic is past oriented.

Let $(E, p, M)$ be a vector bundle over $M, \Omega \subset M$ a domain, $x \in \Omega$ and $V$ a vector space. By $\mathcal{D}^{\prime}\left(\Omega, E^{*} ; V\right)$ we denote the space of distributions on $\left.E^{*}\right|_{\Omega}$ with values in $V$

$$
\mathcal{D}^{\prime}\left(\Omega, E^{*} ; V\right):=\left\{T: \Gamma_{0}\left(\Omega, E^{*}\right) \longrightarrow V \mid T \text { linear and continuous }\right\} .
$$

Each differential operator $P$ on $E$ extends to $\mathcal{D}^{\prime}\left(\Omega, E^{*} ; V\right)$ in the following way. If $P^{*}$ denotes the dual operator on $E^{*}$, defined by

$$
\int_{M} \psi(P \varphi) \mu=\int_{M}\left(P^{*} \psi\right)(\varphi) \mu, \quad \psi \in \Gamma_{0}\left(E^{*}\right), \varphi \in \Gamma_{0}(E),
$$

then for each distribution $T \in \mathcal{D}^{\prime}\left(\Omega, E^{*} ; V\right)$ the distribution $P T$ is defined by

$$
\langle P T, u\rangle:=\left\langle T, P^{*} u\right\rangle \quad u \in \Gamma_{0}\left(\Omega, E^{*}\right) .
$$

A distribution $G \in \mathcal{D}^{\prime}\left(\Omega, E^{*} ; E_{x}^{*}\right)$ is called fundamental solution of $P$ with respect to $(\Omega, x)$, if $P G=\delta_{x}^{E^{*}}$, where $\delta_{x}^{E^{*}}$ is the Dirac distribution of $E_{\Omega}^{*}$ centered at $x$ : $\delta_{x}^{E^{*}}(u):=u(x)$.

In general there exists no fundamental solution of a normally hyperbolic operator $P$ with respect to $(\Omega, x)$. One has to restrict oneself to a certain class of domains $\Omega$, the so called causal domains.

Definition $3 A$ domain $\Omega_{0} \subset M$ is called causal domain if 1. $\Omega_{0}$ is contained in a time and space oriented geodesically normal domain $\Omega$ and 2. $\mathcal{J}_{+}^{\Omega}(x) \cap \mathcal{J}_{-}^{\Omega}(y)$ is compact (or empty) and contained in $\Omega_{0}$ for all $x, y \in \Omega_{0}$.

Proposition 1 ([Fri75], Th.4.4.1): Each Lorentzian manifold can be covered by causal domains.

Definition $4 A$ subset $A \subset \Omega_{0}$ of a causal domain $\Omega_{0}$ is called past compact (or future compact), if $A \cap \mathcal{J}_{-}^{\Omega_{0}}(x)$ (or $A \cap \mathcal{J}_{+}^{\Omega_{0}}(x)$ ) is compact (or empty) for all $x \in \Omega_{0}$.

Notation: $\mathcal{D}_{+}^{\prime}\left(\Omega_{0}, E^{*} ; E_{x}^{*}\right) \subset \mathcal{D}^{\prime}\left(\Omega_{0}, E^{*} ; E_{x}^{*}\right)$ denotes the subset of all distributions with past compact support. $\mathcal{D}_{-}^{\prime}\left(\Omega_{0}, E^{*} ; E_{x}^{*}\right) \subset \mathcal{D}^{\prime}\left(\Omega_{0}, E^{*} ; E_{x}^{*}\right)$ denotes the subset of all distributions with future compact support.

Theorem 2 (GGün88], chap.3.3): Let $P: \Gamma(E) \longrightarrow \Gamma(E)$ be a normally hyperbolic operator, let $\Omega_{0} \subset M$ be a causal domain and $x \in \Omega_{0}$. Then there exists exactly one fundamental solution $G_{+}^{\Omega_{0}}(x) \in \mathcal{D}_{+}^{\prime}\left(\Omega_{0}, E^{*} ; E_{x}^{*}\right)$ of $P$ with respect to $\left(\Omega_{0}, x\right)$ with past 
compact support (forward fundamental solution) and there exists exactly one fundamental solution $G_{-}^{\Omega_{0}}(x) \in \mathcal{D}_{-}^{\prime}\left(\Omega_{0}, E^{*} ; E_{x}^{*}\right)$ of $P$ with respect to $\left(\Omega_{0}, x\right)$ with future compact support (backward fundamental solution).

The support and singular support of these fundamental solutions satisfy

$$
\begin{array}{rll}
\operatorname{supp} G_{ \pm}^{\Omega_{0}}(x) & \subset & \mathcal{J}_{ \pm}^{\Omega_{0}}(x) \\
\operatorname{singsupp} G_{ \pm}^{\Omega_{0}}(x) & \subset & \mathcal{C}_{ \pm}^{\Omega_{0}}(x) .
\end{array}
$$

Now we can define the notion of a Huygens operator.

Definition $5 P$ is called a Huygens operator or an operator of Huygens type if there exists a covering $\mathcal{U}$ of $M$ by causal domains such that for each causal domain $\Omega_{0} \in \mathcal{U}$ and each $x \in \Omega_{0}$ the forward and backward fundamental solution $G_{ \pm}^{\Omega_{0}}(x)$ of $P$ with respect to $\left(\Omega_{0}, x\right)$ is supported in the light cone $\mathcal{C}_{ \pm}^{\Omega_{0}}(x)$.

From the structure of the fundamental solutions it is easy to see, that a normally hyperbolic operator on a manifold of odd dimension or dimension 2 can never be of Huygens type (see [Gün88], chap.3.3).

Analytic properties of a Huygens operator resulting from the special property of its fundamental solutions can be found in [Gün88], chap.4. We will make use of the so-called Hadamard criterion, which says that an operator $P$ is of Huygens type if a certain Hadamard coefficients associated to $P$ vanishes. To make this statement more precise, we will now recall the definition of the Hadamard coefficients of a normally hyperbolic operator.

Let $\nabla$ be a covariant derivative on a vector bundle $E$ over a Lorentzian manifold $(M, g)$. Let us denote by $\nabla^{T^{*} M \otimes E}$ the covariant derivative defined by the Levi-Civita connection of $(M, g)$ and $\nabla$. The operator

$$
\triangle^{\nabla}:=-\operatorname{trace}_{g}\left(\nabla^{T^{*} M \otimes E} \circ \nabla\right)
$$

is called Bochner-Laplace operator defined by $\nabla$.

Proposition 2 Let $P: \Gamma(E) \longrightarrow \Gamma(E)$ be a normally hyperbolic operator on $E$ and let $g$ be the Lorentzian metric given by the principal symbol of $P$. Then there exists a uniquely determined covariant derivative $\nabla^{P}: \Gamma(E) \longrightarrow \Gamma\left(T^{*} M \otimes E\right)$ and a homomorphism $H_{P} \in \Gamma(H o m(E, E))$ such that

$$
P:=\triangle^{P}+H_{P} .
$$

(1) is called Weitzenböck formula for $P$.

For two vector bundles $E$ and $F$ over $M^{n}$ we denote by $E \otimes F$ the external tensor product of these bundles over $M \times M$ :

$$
E \otimes F:=p r_{1}^{*} E \otimes p r_{2}^{*} F .
$$


We often identify the fibre of $E^{*} \otimes E$ over a point $(x, y) \in M \times M$ with the set of homomorphisms $\operatorname{Hom}\left(E_{x}, E_{y}\right)$. Let $\Omega \subset M$ be a geodesically normal domain in $M$ and let $\sigma: \Omega \times \Omega \longrightarrow \mathbb{R}$ be the quadratic geodesic distance function on $\Omega$. The function $\sigma_{x}: \Omega \longrightarrow \mathbb{R}$ is given by $\sigma_{x}(y):=\sigma(x, y)$. The function $m \in C^{\infty}(\Omega \times \Omega)$

$$
m(x, \cdot):=-\frac{1}{2} \Delta \sigma_{x}-n
$$

is called divergence measure of $\Omega$. By $\tau \in C^{\infty}(\Omega \times \Omega)$ we denote the function

$$
\tau(x, y):=\exp \left\{\frac{1}{2} \int_{0}^{1} \frac{m(x, \gamma(s))}{s} d s\right\},
$$

where $\gamma:[0,1] \longrightarrow \Omega$ denotes the unique geodesic in $\Omega$ joining $x$ and $y$.

Proposition 3 Let $P: \Gamma(E) \longrightarrow \Gamma(E)$ be a normally hyperbolic operator and let $\Omega \subset M$ be a geodesically normal domain. Then there exists a uniquely determined sequence of sections $U_{k} \in \Gamma\left(\Omega \times \Omega, E^{*} \otimes E\right), k=0,1,2, \ldots$, such that the following differential equations and initial conditions are satisfied on $\Omega$ for all $x \in \Omega$ :

$$
\begin{aligned}
\nabla_{g r a d \sigma_{x}}^{P} U_{k}(x, \cdot)+(m(x, \cdot) & +2 k) U_{k}(x, \cdot)=-P\left(U_{k-1}(x, \cdot)\right) \\
U_{-1} & =0 \\
U_{0}(x, x) & =I d_{E_{x}} .
\end{aligned}
$$

Let us denote by $\mathcal{P}(x, y) \in \operatorname{Hom}\left(E_{x}, E_{y}\right)$ the parallel displacement along the geodesic $\gamma$ joining $x$ and $y$ in $\Omega$. Then the sections $U_{k}$ satisfy

$$
\begin{aligned}
U_{0}(x, y) & =\frac{1}{\tau(x, y)} \mathcal{P}(x, y) \\
U_{k}(x, y) & =-\frac{1}{2 \tau(x, y)} \int_{0}^{1} t^{1-k} \tau(x, \gamma(t)) \mathcal{P}(\gamma(t), y) P\left(U_{k-1}(x, \cdot)\right)(\gamma(t)) d t \quad k \geq 1
\end{aligned}
$$

All differentiations refer to the second component.

The sections $U_{k} \in \Gamma\left(\Omega \times \Omega, E^{*} \otimes E\right), k=0,1,2, \ldots$, are called Hadamard coefficients of $P$ over $\Omega$. Then the Hadamard criterion says

Theorem 3 (Hadamard Criterion) Let $P: \Gamma(E) \longrightarrow \Gamma(E)$ be a normally hyperbolic operator over a manifold $M$ of even dimension $n \geq 4$. Then $P$ is a Huygens operator if and only if there exists a covering $\mathcal{U}$ by causal domains such that the Hadamard coefficient $U_{\frac{n-2}{2}}$ of $P$ satisfies

$$
U_{\frac{n-2}{2}}(x, y)=0
$$

for all $\Omega_{0} \in \mathcal{U}, x \in \Omega_{0}, y \in \mathcal{C}^{\Omega_{0}}(x)$. 
Another method to find Huygens operators is founded on the conformal gauge invariance of the Huygens property. Let $M^{n}$ be a manifold of even dimension $n \geq 4$ and let $E$ be a vector bundle over $M^{n}$. To each normally hyperbolic operator $P: \Gamma(E) \longrightarrow \Gamma(E)$ is assigned a sequence $I_{k}(P) \in \Gamma\left(S^{k}\left(T^{*} M\right) \otimes \operatorname{Hom}(E, E)\right), k=0,1,2, \ldots$, of symmetric trace free conformal gauge invariants of weight $\omega=1-\frac{n}{2}$, the so-called moments of $P$ of order $k$ (see [Gün88], chap.6). If $P$ is a Huygens operator, then all moments $I_{k}(P), k=0,1,2, \ldots$, vanish. Moreover, if $M$ and $P$ are analytic the vanishing of all moments imply the Huygens property for $P$. In dimension $n=4$ there are explicit formulas which express the moments of order $\leq 4$ in terms of the curvature of the manifold $\left(M^{4}, g\right)$ and the curvature of the covariant derivative $\nabla^{P}$ associated to $P$ by its Weitzenböck formula (see [Gün88], chap.7, Table II). Using these formulas for the first three moments one obtains

Theorem 4 Let $P: \Gamma(E) \longrightarrow \Gamma(E)$ be a normally huyperbolic operator of Huygens type on a 4-dimensional manifold $M^{4}$. Let $g$ be the Lorentzian metric defined by $P, R$ the scalar curvature of $\left(M^{4}, g\right), \nabla^{P}$ the covariant derivative and $H_{P}$ the homomorphism on $E$ associated to $P$ by its Weitzenböck formula. Then the following conditions are satisfied:

1. The Cotton invariant $C_{P}:=H_{P}-\frac{1}{6} R$ of $P$ vanishes.

2. $\nabla^{P}$ is a Yang-Mills connection: for the curvature $F^{P}$ of $\nabla^{P} \quad \delta F^{P}=0$ holds.

3. The Bach tensor $\mathcal{B}$ of $\left(M^{4}, g\right)$ equals a multiple of the "energy impuls tensor" of $F^{P}$ :

$$
\mathcal{B} \otimes I d_{E}=-5\left(Q_{2}\left(F^{P}, F^{P}\right)-\frac{1}{4} g \otimes\left\|F^{P}\right\|^{2}\right) .
$$

\section{The Dirac operator of Lorentzian space forms}

Let $\left(M^{n}, g\right)$ be a Lorentzian spin manifold of constant sectional curvature $K$ and even dimension $n \geq 4$. Let us denote by $D: \Gamma(S) \longrightarrow \Gamma(S)$ the Dirac operator of $\left(M^{n}, g\right)$. In this chapter we will prove, that the operator $D^{2}-K: \Gamma(S) \longrightarrow \Gamma(S)$ is of Huygens type.

The proof is based on the fact, that the spinor bundle over a simply connected semiRiemannian space form can be trivialized by Killing spinors and on the fact, that the Yamabe operator of a conformally flat Lorentzian manifold is of Huygens type (see [Gün88], chap.6.3).

Definition 6 Let $\left(M^{n}, g\right)$ be a Lorentzian spin manifold with spinor bundle $S . A$ spinor field $\varphi \in \Gamma(S)$ is called a Killing spinor to the Killing number $\lambda \in \mathbb{C}$ if

$$
\nabla_{X}^{S} \varphi=\lambda X \cdot \varphi
$$

for all vector fields $X$ on $M$. 
Let us denote by $M_{K}^{n}$ the simply connected Lorentzian manifold of constant sectional curvature $K$. Since $M_{K}^{n}$ is parallelizable it is orientable and has exactly one spinor structure (the trivial spinor structure). Let $S$ be the spinor bundle of $M_{K}^{n}$.

Proposition 4 ([CGLS86]) The spinor bundle $S$ on $M_{K}^{n}$ can be trivialized by Killing spinors to the Killing number $\mu \in \mathbb{C}$, where $\mu^{2}=\frac{K}{4}$.

Proof: Let $\mu \in \mathbb{C}$ be a complex number with $\mu^{2}=\frac{K}{4}$ and let denote $\nabla^{\mu}$ the covariant derivative

$$
\nabla_{X}^{\mu}:=\nabla_{X}^{S}-\mu X
$$

on $S$. A Killing spinor to the Killing number $\mu$ is parallel with respect to $\nabla^{\mu}$. Since $M_{K}^{n}$ is simply connected it is enough to prove that the curvature $F^{\mu}$ of $\nabla^{\mu}$ vanishes on the bundle $S$. Now, it is easy to check that

$$
\begin{aligned}
F^{\mu}(X, Y) & =\nabla_{X}^{\mu} \nabla_{Y}^{\mu}-\nabla_{Y}^{\mu} \nabla_{X}^{\mu}-\nabla_{[X, Y]}^{\mu} \\
& =F^{S}(X, Y)+\mu^{2}(X \cdot Y-Y \cdot X) .
\end{aligned}
$$

The curvature $F^{S}$ of the spinor derivative $\nabla^{S}$ is given by

$$
F^{S}(X, Y)=\frac{1}{4} \sum_{i j} \varepsilon_{i} \varepsilon_{j} \mathcal{R}\left(X, Y, s_{i}, s_{j}\right) s_{i} \cdot s_{j}
$$

where $\mathcal{R}$ denotes the curvature tensor of the basis manifold and $\left(s_{1}, \ldots, s_{n}\right)$ is a local orthonormal basis. The curvature tensor of a manifold of constant sectional curvature $K$ is given by

$$
\mathcal{R}(X, Y, V, W)=K\{g(X, W) g(Y, V)-g(X, V) g(Y, W)\} .
$$

Hence on $M_{K}^{n}$ we have for the curvature of the spinor derivative

$$
F^{S}(X, Y)=\frac{1}{4} K(Y \cdot X-X \cdot Y) .
$$

Since $\mu^{2}=\frac{1}{4} K$ from (2) it follows that $F^{\mu} \equiv 0$ on $S$.

Helgason ([Hel94], chap.5.5.4) and Schimming and Schlichtkrull ([SS94]) proved the following Theorem for the Laplace operator of a Lorentzian space form

Theorem 5 Let $\left(M^{n}, g\right)$ be a Lorentzian manifold of constant sectional curvature $K$ and even dimension $n \geq 4$. Then for each $m=3,5, \ldots, n-1$ the shifted Laplace operator

$$
L_{m}:=\Delta_{0}+K(n-m)(m-1)
$$

is of Huygens type. 
This can be used to prove

Theorem 6 Let $\left(M^{n}, g\right)$ be a Lorentzian spin manifold of constant sectional curvature $K$ and even dimension $n \geq 4$ and denote by $D$ the Dirac operator of $\left(M^{n}, g\right)$. Let $\mu \in \mathbb{C}$ be a complex number with $\mu^{2}=\frac{K}{4}$. Then for each $m=3,5, \ldots, n-1$ the operators

$$
\begin{aligned}
& P_{m,-}:=(D-\mu)^{2}-\frac{K}{4}\left\{(n-1)^{2}-4(n-m)(m-1)\right\} \quad \text { and } \\
& P_{m,+}:=(D+\mu)^{2}-\frac{K}{4}\left\{(n-1)^{2}-4(n-m)(m-1)\right\}
\end{aligned}
$$

are of Huygens type.

Proof: Let $\lambda \in \mathbb{C}$ be a complex number. The Weitzenböck formula of $(D-\lambda)^{2}$ is

$$
(D-\lambda)^{2}=\Delta^{\lambda}+\frac{1}{4} n(n-1) K+(1-n) \lambda^{2}
$$

where $\Delta^{\lambda}$ is the Bochner-Laplace operator of the covariant derivative $\nabla^{\lambda}$ defined by $\nabla_{X}^{\lambda}:=\nabla_{X}^{S}-\lambda X$

$$
\Delta^{\lambda}=-\sum_{i=1}^{n} \varepsilon_{i}\left(\nabla_{s_{i}}^{\lambda} \nabla_{s_{i}}^{\lambda}+\operatorname{div}\left(s_{i}\right) \nabla_{s_{i}}^{\lambda}\right)
$$

For a function $f \in C^{\infty}(M)$ and a spinor field $\psi \in \Gamma(S)$ we have

$$
\Delta^{\lambda}(f \psi)=\Delta_{0}(f) \psi+f \Delta^{\lambda} \psi-2 \nabla_{g r a d}^{\lambda}(f) \psi
$$

Hence, if $\varphi$ is a Killing spinor to the Killing number $\mu$ with $\mu^{2}=\frac{1}{4} K$, then from (3) and (4) it follows that

$$
(D-\mu)^{2}(f \varphi)=\Delta_{0}(f) \varphi+\frac{K}{4}(n-1)^{2} f \varphi .
$$

Since the Huygens property in a local one, it is enough to prove the Theorem for the simply connected manifold $M_{K}^{n}$. According to Proposition 4 there exists a basis of Killing spinors $\varphi_{1}, \ldots, \varphi_{N}$ to the Killing number $\mu$ in the spinor bundle $S$ of $M_{K}^{n}$. Hence the space of spinor fields $\Gamma\left(M_{K}^{n}, S\right)$ on $M_{K}^{n}$ can be identified with that of smooth functions $C^{\infty}\left(M_{K}^{n}, \mathbb{C}^{N}\right)$ by assigning to each spinor field $\varphi=\sum_{\varepsilon=1}^{N} f_{\varepsilon} \varphi_{\varepsilon}$ the function $\left(f_{1}, \ldots, f_{N}\right)$. Using this identification according to $(5)$ the operator $(D-\mu)^{2}$ corresponds to the operator $\Delta_{0}+\frac{K}{4}(n-1)^{2}$. Then from Theorem 5 it follows that for each $m=3,5, \ldots, n-1$ the operator

$$
P_{m,-}=(D-\mu)^{2}-\frac{K}{4}\left\{(n-1)^{2}-4(n-m)(m-1)\right\}
$$

acting on $\Gamma\left(M_{K}^{n}, S\right)$ is of Huygens type. If we trivialize the spinor bundle of $M_{K}^{n}$ using Killing spinors to the Killing number $-\mu$ we obtain in the same manner the result for the operator $P_{m,+}=(D+\mu)^{2}-\frac{K}{4}\left\{(n-1)^{2}-4(n-m)(m-1)\right\}$. 
Now, we are able to prove the above mentioned result:

Theorem 7 Let $\left(M^{n}, g\right)$ be a Lorentzian spin manifold of constant sectional curvature $K$ and even dimension $n \geq 4$ and let us denote by $D$ the Dirac operator of $\left(M^{n}, g\right)$. Then the operator $D^{2}-K: \Gamma(S) \longrightarrow \Gamma(S)$ is of Huygens type.

Proof: If $K$ is zero, the theorem follows from Theorem 6 . Hence we assume, that $K \neq 0$. Let $\mu$ be a complex number with $\mu^{2}=\frac{K}{4}$ and let $m=2\left[\frac{n}{4}\right]+1$. According to Theorem 6 the operators $P_{m_{1}-}=(D-\mu)^{2}-\mu^{2}=(D-2 \mu) D$ and $P_{m,+}=$ $(D+\mu)^{2}-\mu^{2}=(D+2 \mu) D$ are Huygens operators. (In the simply connected case, these operators correspond to the Yamabe operator acting on $C^{\infty}\left(M_{K}^{n}, \mathbb{C}^{N}\right)$, if we trivialize the spinor bundle $S$ using Killing spinors to the Killing number $\mu$ and $-\mu$, respectively). Since $(D-2 \mu) D$ and $(D+2 \mu) D$ are of Huygens type, there exists a covering $\mathcal{U}$ by causal domains such that for all $\Omega_{0} \in \mathcal{U}$ and $x \in \Omega_{0}$ the forward and backward fundamental solutions $G_{1, \pm}^{\Omega_{0}}(x)$ and $G_{2, \pm}^{\Omega_{0}}(x)$ of $(D-2 \mu) D$ and $(D+$ $2 \mu) D$, respectively, are supported in the light cone $C_{ \pm}^{\Omega_{0}}(x)$. Consider the distributions $H_{1, \pm}^{\Omega_{0}}(x):=D G_{1, \pm}(x)$ and $H_{2, \pm}^{\Omega_{0}}(x):=D G_{2, \pm}(x)$. Then

$$
\begin{gathered}
(D+2 \mu)(D-2 \mu) H_{1, \pm}^{\Omega_{0}}(x)=(D+2 \mu) \delta_{x}=D \delta_{x}+2 \mu \delta_{x} \\
(D-2 \mu)(D+2 \mu) H_{2, \pm}^{\Omega_{0}}(x)=(D-2 \mu) \delta_{x}=D \delta_{x}-2 \mu \delta_{x} .
\end{gathered}
$$

Hence

$$
\left(D^{2}-4 \mu^{2}\right)\left(\frac{1}{4 \mu}\left[H_{1, \pm}^{\Omega_{0}}(x)-H_{2, \pm}^{\Omega_{0}}(x)\right]\right)=\delta_{x} .
$$

Therefore, the distribution $E_{ \pm}^{\Omega_{0}}(x) \in \mathcal{D}_{ \pm}^{\prime}\left(\Omega_{0}, S^{*} ; S_{x}\right)$ defined by

$$
E_{ \pm}^{\Omega_{0}}(x):=\frac{1}{4 \mu}\left[H_{1, \pm}^{\Omega_{0}}(x)-H_{2, \pm}^{\Omega_{0}}(x)\right]
$$

is the forward resp. backward fundamental solution of the operator $D^{2}-K$ with respect to $\left(\Omega_{0}, x\right)$. Since

$$
\begin{aligned}
\operatorname{supp} E_{ \pm}^{\Omega_{0}}(x) & \subset \operatorname{supp} H_{1, \pm}^{\Omega_{0}}(x) \cup \operatorname{supp} H_{2, \pm}^{\Omega_{0}}(x) \\
& \subset \operatorname{supp} G_{1, \pm}^{\Omega_{0}}(x) \cup \operatorname{supp} G_{2, \pm}^{\Omega_{0}}(x) \\
& \subset C_{ \pm}^{\Omega_{0}}(x),
\end{aligned}
$$

$D^{2}-K$ is a Huygens operator.

\section{The Dirac operator on plane wave manifolds}

Already for a long time it is known that the Laplace-Beltrami operator on a plane wave manifold of even dimension $n \geq 4$ and the Hodge-Laplace operators on forms of a plane wave manifold of even dimension $n \geq 6$ are of Huygens type (see [Gün65], [Sch71], [Gün88]). In this section we prove, that the square of the Dirac operator on plane wave spin manifolds of even dimension $n \geq 4$ is a Huygens operator. 
Definition 7 A Lorentzian manifold $\left(M^{n}, g\right)$ is called a plane wave manifold if the following conditions are satisfied:

1. There exists an isotropic parallel vector field $T$ on $M$.

2. For the curvature tensor $\mathcal{R}$ of $(M, g)$

$$
\operatorname{trace}_{(3,5),(4,6)} \mathcal{R} \otimes \mathcal{R}=0
$$

holds, where trace ${ }_{(i, j)} B$ denotes the trace of the tensor field $B$ in the $i$-th and $j$-th component with respect to $g$.

3. $\mathcal{R}$ is quasi recurrent with $T$ i.e. that there exists a $(4,0)$-tensor field $\mathcal{R}_{1}$ such that

$$
\nabla \mathcal{R}=T^{\mathrm{b}} \otimes \mathcal{R}_{1}
$$

where $T^{b}$ denotes the 1 -form dual to $T$ with respect to $g$.

A plane wave manifold is foliated by submanifolds of codimension 1, the integral curves of $T$ are isotropic geodesics running in the leaves of the foliation and $M$ is locally symmetric along the leaves: $\nabla_{X} \mathcal{R}=0$ for all vectors $X$ tangent to the leaves of the foliation. The scalar curvature of a plane wave manifold is zero.

The geometry of plane wave manifolds was studied by Schimming in [Sch74].

Theorem 8 ([Sch74]) A Lorentzian manifold $\left(M^{n}, g\right)$ is a plane wave manifold with the isotropic parallel vector field $T$ if and only if for each point $x \in M$ there exists a coordinate neighbourhood $\left(U,\left(x^{1}, \ldots, x^{n}\right)\right)$ such that the metric $g$ has the form

$$
g_{\mid U}=2 d x^{1} d x^{2}+a_{\alpha \beta}\left(x^{1}\right) d x^{\alpha} d x^{\beta} \quad 3 \leq \alpha, \beta \leq n
$$

where $\left(a_{\alpha \beta}\right)$ is a positive definite matrix, depending only on $x^{1}$ and the vector field $T$ is given by $T_{\mid U}=\frac{\partial}{\partial x^{2}}$.

\subsection{Some geometry of standard plane wave manifolds}

In this section we consider the following "standard" plane wave manifold $(M, g)$ :

Let $M:=I \times \mathbb{R}^{n-1}$ be the product of an open interval $I$ with the $\mathbb{R}^{n-1}$ and $g$ be the Lorentzian metric

$$
g=2 d x_{1} d x_{2}+d \widetilde{x} A\left(x_{1}\right) d \widetilde{x}^{t}
$$

where $x=\left(x_{1}, \ldots, x_{n}\right) \in M, \tilde{x}=\left(x_{3}, \ldots, x_{n}\right)$ and $A\left(x_{1}\right)$ denotes a positive definite $(n-2) \times(n-2)$-matrix, depending smoothly on $x_{1}$. Then $T:=\frac{\partial}{\partial x_{2}}$ is the isotropic, parallel vector field occuring in the definition of a plane wave manifold. We will explain some geometric properties of standard plane wave manifolds which we need to calculate the Hadamard coefficients of the square of the Dirac operator.

With small letters $i, j, k, l, \ldots$ we will denote indices running from $1, \ldots$, n. With capital letters $I, J, K, L, \ldots$ we will denote indices running from $3, \ldots$, n. $\left(\partial_{1}, \ldots, \partial_{n}\right)$ is the canonical basis with respect to the coordinates $\left(x_{1}, \ldots, x_{n}\right)$.

We will use the following denotations: 
Definition 8 Let $A$ be the matrix occuring in the definition of the metric $g$. Then $V$ and $W$ are the symmetric matrices:

$$
\begin{aligned}
V\left(q_{1}, q_{2}\right) & :=\int_{0}^{1} A^{-1}\left(q_{1}+t\left(q_{2}-q_{1}\right)\right) d t \\
W\left(q_{1}, q_{2}\right) & :=\left(V\left(q_{1}, q_{2}\right)\right)^{-1} .
\end{aligned}
$$

By a simple calculation it follows

Lemma 1 The only non-vanishing Christoffel symbols of $(M, g)$ with respect to the coordinates $\left(x_{1}, \ldots, x_{n}\right)$ are

$$
\begin{array}{rr}
\Gamma_{I I}^{K}=\Gamma_{I 1}^{K}=\frac{1}{2}\left(A^{-1} \dot{A}\right)_{K I} & I, K=3, \ldots, n \\
\Gamma_{I J}^{2}=-\frac{1}{2} \dot{A}_{I J} & I, J,=3, \ldots, n
\end{array}
$$

where $\dot{A}$ denotes the derivative of $A$ with respect to $x_{1}$.

The geodesics of the standard plane wave manifold $(M, g)$ are described in the following proposition.

Proposition 5 Let $y, z$ be two points of $M$. Then there is exactly one geodesic $\gamma=$ $\left(\gamma_{1}, \gamma_{2}, \tilde{\gamma}\right):[0,1] \longrightarrow M$ joining $y$ with $z$.

This geodesic is given by

$$
\begin{aligned}
& \gamma_{1}(s)=y_{1}+\left(z_{1}-y_{1}\right) s \\
& \gamma_{2}(s)= \begin{cases}y_{2}+\left(z_{2}-y_{2}\right) s+\frac{s}{2\left(z_{1}-y_{1}\right)}(\tilde{z}-\tilde{y})[W-W V(s) W](\tilde{z}-\tilde{y})^{t} & \text { if } z_{1} \neq y_{1} \\
y_{2}+\left(z_{2}-y_{2}\right) s+\frac{1}{4}\left(s^{2}-s\right)(\tilde{z}-\tilde{y}) \dot{A}\left(y_{1}\right)(\tilde{z}-\tilde{y})^{t} & \text { if } z_{1}=y_{1}\end{cases} \\
& \widetilde{\gamma}(s)^{t}=\widetilde{y}^{t}+s V(s) W(\widetilde{z}-\widetilde{y})^{t}
\end{aligned}
$$

where $V(s)$ and $W$ are the matrices

$$
V(s):=V\left(y_{1}, \gamma_{1}(s)\right) \quad W:=W\left(y_{1}, z_{1}\right) .
$$

In particular, $M$ is a geodesically normal domain.

Proof: Let $\delta(s)=\left(\delta_{1}(s), \delta_{2}(s), \tilde{\delta}(s)\right)$ be the geodesic with $\delta(0)=y=\left(y_{1}, y_{2}, \widetilde{y}\right)$, $\delta^{\prime}(0)=v=\left(v_{1}, v_{2}, \widetilde{v}\right)$. Using Lemma 1 , the geodesic equations

$$
\delta_{k}^{\prime \prime}(s)+\sum_{i j} \delta_{i}^{\prime}(s) \delta_{j}^{\prime}(s) \Gamma_{i j}^{k}(\delta(s))=0
$$

result

$$
\begin{aligned}
\delta_{1}^{\prime \prime}(s) & =0 \\
\delta_{2}^{\prime \prime}(s) & =\frac{1}{2} \tilde{\delta}^{\prime}(s) \dot{A}\left(\delta_{1}(s)\right) \tilde{\delta}^{\prime}(s)^{t} \\
\tilde{\delta}^{\prime \prime}(s)^{t} & =-\delta_{1}^{\prime}(s) A^{-1}\left(\delta_{1}(s)\right) \dot{A}\left(\delta_{1}(s)\right) \tilde{\delta}^{\prime}(s)^{t}
\end{aligned}
$$


Hence,

$$
\delta_{1}(s)=y_{1}+v_{1} s
$$

Let $\mathcal{A}(s):=A\left(\delta_{1}(s)\right)$. Using $\mathcal{A}^{\prime}(s)=\dot{A}\left(\delta_{1}(s)\right) \delta_{1}^{\prime}(s)$ we obtain from (8)

$$
\mathcal{A}(s) \tilde{\delta}^{\prime \prime}(s)^{t}+\mathcal{A}^{\prime}(s) \widetilde{\delta}^{\prime}(s)^{t}=\left(\mathcal{A} \tilde{\delta}^{\prime \prime}\right)^{\prime}(s)=0 .
$$

From the initial data it follows

$$
\begin{aligned}
\tilde{\delta}(s)^{t} & =\left(\int_{0}^{s} \mathcal{A}^{-1}(t) d t\right) A\left(y_{1}\right) \widetilde{v}^{t}+\widetilde{y}^{t} \\
& =\mathcal{V}(s) A\left(y_{1}\right) \widetilde{v}^{t} s+\widetilde{y}^{t}
\end{aligned}
$$

where $\mathcal{V}(s):=V\left(y_{1}, \delta_{1}(s)\right)$.

Now, let $v_{1} \neq 0$. Then (7) and (10) yield

$$
\delta_{2}^{\prime \prime}(s)=-\frac{1}{2 v_{1}} \widetilde{v} A\left(y_{1}\right)\left(\mathcal{A}^{-1}\right)^{\prime}(s) A\left(y_{1}\right) \widetilde{v}^{t}
$$

Hence,

$$
\delta_{2}(s)=y_{2}+v_{2} s+\frac{s}{2 v_{1}} \tilde{v}\left[A\left(y_{1}\right)-A\left(y_{1}\right) \mathcal{V}(s) A\left(y_{1}\right)\right] \widetilde{v}^{t}
$$

In case $v_{1}=0$, from $\delta_{1}(s)=y_{1}$ it follow $\mathcal{A}(s) \equiv A\left(y_{1}\right)$ and $\mathcal{V}(s) \equiv A^{-1}\left(y_{1}\right)$. Therefore, (10) gives

$$
\widetilde{\delta}(s)=\widetilde{y}+\widetilde{v} s
$$

and (7) results

$$
\delta_{2}(s)=y_{2}+v_{2} s+\frac{1}{4} \tilde{v} \dot{A}\left(y_{1}\right) \tilde{v}^{t} s^{2} .
$$

Now, let $\gamma:[0,1] \longrightarrow M$ be a geodesic in $M$ joining $y$ with $z$ and let $v \in T_{y} M$ be the vector $v=\gamma^{\prime}(0)$. Then with $\gamma(s)=\delta(s)$ the formulas $(9),(10),(11)$ and (12) show that $v$ is uniquely determined by $z=\delta(1)$.

We obtain

$$
\begin{aligned}
& v_{1}=z_{1}-y_{1} \\
& v_{2}= \begin{cases}z_{2}-y_{2}-\frac{1}{2\left(z_{1}-y_{1}\right)}(\tilde{z}-\tilde{y})\left[W A^{-1}\left(y_{1}\right) W-W\right](\tilde{z}-\tilde{y})^{t} & \text { if } z_{1} \neq y_{1} \\
z_{2}-y_{2}-\frac{1}{4}(\tilde{z}-\tilde{y}) \dot{A}\left(y_{1}\right)(\widetilde{z}-\widetilde{y})^{t} & \text { if } z_{1}=y_{1}\end{cases} \\
& \tilde{v}^{t}=A^{-1}\left(y_{1}\right) W(\widetilde{z}-\tilde{y})^{t}
\end{aligned}
$$

Inserting this in (9), (10), (11) and (12) the proposition is proved.

Now, we can calculate the quadratic geodesic distance function and the divergence measure of $(M, g)$ : 
Proposition 6 The quadratic geodesic distance function $\sigma: M \times M \longrightarrow \mathbb{R}$ of the standard plane wave manifold $(M, g)$ is given by

$$
\sigma(y, z)=2\left(z_{1}-y_{1}\right)\left(z_{2}-y_{2}\right)+(\tilde{z}-\tilde{y}) W\left(y_{1}, z_{1}\right)(\tilde{z}-\widetilde{y})^{t} .
$$

Proof: Let $\gamma:[0,1] \longrightarrow M$ be the geodesic joining $y$ with $z$ and let $v=\gamma^{\prime}(0)$. By definition

$$
\begin{aligned}
\sigma(y, z) & =g\left(\gamma^{\prime}(s), \gamma^{\prime}(s)\right) \equiv g\left(\gamma^{\prime}(0), \gamma^{\prime}(0)\right) \\
& =2 v_{1} v_{2}+\widetilde{v} A\left(y_{1}\right) \tilde{v}^{t}
\end{aligned}
$$

Inserting (13), (14), (15) and regard $W\left(y_{1}, y_{1}\right)=A\left(y_{1}\right)$ we obtain the assertion.

Proposition 7 The divergence measure of the standard plane wave manifold $\left(M^{n}, g\right)$ is given by

$$
m(y, z)=-\left(z_{1}-y_{1}\right)\left(\ln \frac{w_{y_{1}}}{\sqrt{a}}\right)^{\prime}\left(z_{1}\right)
$$

where $w_{y_{1}}\left(z_{1}\right):=w\left(y_{1}, z_{1}\right):=\operatorname{det}\left[W\left(y_{1}, z_{1}\right)\right], a\left(z_{1}\right):=\operatorname{det}\left[A\left(z_{1}\right)\right]$.

Proof: Let $\sigma_{y}(z):=\sigma(y, z)$ and $W_{y_{1}}\left(z_{1}\right)=W\left(y_{1}, z_{1}\right)$. By definition the divergence measure $m(y, z)$ is

$$
m(y, \cdot)=-\frac{1}{2} \Delta\left(\sigma_{y}\right)-n .
$$

An easy calculation shows that the Laplacian $\Delta$ of a function $u \in C^{\infty}(M)$ is

$$
\Delta u(x)=-2 \frac{\partial^{2} u}{\partial x_{1} \partial x_{2}}(x)-A\left(x_{1}\right)^{I J} \frac{\partial^{2} u}{\partial x_{I} \partial x_{J}}(x)-(\ln \sqrt{a})^{\prime}\left(x_{1}\right) \frac{\partial u}{\partial x_{2}}(x)
$$

where $\left(A^{I J}\left(x_{1}\right)\right)$ denote the elements of the inverse matrix $A^{-1}\left(x_{1}\right)$. Using Proposition 6 we obtain

$$
\Delta\left(\sigma_{y}\right)(z)=-4-2 \operatorname{trace}\left(A^{-1} W_{y_{1}}\right)\left(z_{1}\right)-2\left(z_{1}-y_{1}\right)(\ln \sqrt{a})^{\prime}\left(z_{1}\right) .
$$

Since trace $\left(W_{y_{1}}^{-1} W_{y_{1}}\right)=n-2$ we can deduce

$$
m(y, z)=\operatorname{trace}\left[\left(A^{-1}-W_{y_{1}}^{-1}\right) W_{y_{1}}\right]\left(z_{1}\right)+\left(z_{1}-y_{1}\right)(\ln \sqrt{a})^{\prime}\left(z_{1}\right) .
$$

From

$$
W^{-1}\left(y_{1}, z_{1}\right)=\int_{0}^{1} A^{-1}\left(y_{1}+t\left(z_{1}-y_{1}\right)\right) d t
$$

it follows

$$
A^{-1}\left(z_{1}\right)-W_{y_{1}}^{-1}\left(z_{1}\right)=\left(W_{y_{1}}^{-1}\right)^{\prime}\left(z_{1}\right)\left(z_{1}-y_{1}\right)
$$

Hence,

$$
m(y, z)=\left(z_{1}-y_{1}\right) \operatorname{trace}\left[\left(W_{y_{1}}^{-1}\right)^{\prime} W_{y_{1}}\right]\left(z_{1}\right)+\left(z_{1}-y_{1}\right)(\ln \sqrt{a})^{\prime}\left(z_{1}\right) .
$$


For a symmetric positive definite matrix $U(t)$

$$
(\ln \operatorname{det}(U))^{\prime}=\operatorname{trace}\left[U^{-1} U^{\prime}\right]=-\operatorname{trace}\left[\left(U^{-1}\right)^{\prime} U\right]
$$

holds. Therefore (16) gives

$$
m(y, z)=-\left(z_{1}-y_{1}\right)\left(\ln \left(\frac{w_{y_{1}}}{\sqrt{a}}\right)\right)^{\prime}\left(z_{1}\right) .
$$

Let $\tau \in C^{\infty}(M \times M)$ be the function

$$
\tau(y, z):=\exp \left\{\frac{1}{2} \int_{0}^{1} \frac{m(y, \gamma(s))}{s} d s\right\}
$$

where $\gamma:[0,1] \longrightarrow M$ denotes the unique geodesic joining $y$ with $z$.

Proposition 8 The function $\tau$ is given by

$$
\tau(y, z)=\frac{\sqrt[4]{a\left(y_{1}\right) a\left(z_{1}\right)}}{\sqrt{w\left(y_{1}, z_{1}\right)}}
$$

Proof: From Proposition 5 we know

$$
\gamma_{1}(s)=y_{1}+\left(z_{1}-y_{1}\right) s .
$$

Using Proposition 7 we obtain

$$
\begin{aligned}
m(y, \gamma(s)) & =-\left(z_{1}-y_{1}\right) s\left(\ln \frac{w_{y_{1}}}{\sqrt{a}}\right)^{\prime}\left(\gamma_{1}(s)\right) \\
& =-s \frac{d}{d s}\left(\ln \frac{w\left(y_{1}, \gamma_{1}(s)\right)}{\sqrt{a\left(\gamma_{1}(s)\right)}}\right)
\end{aligned}
$$

It follows

$$
\tau(y, z)=\exp \left\{\frac{1}{2}\left[\ln \frac{w\left(y_{1}, y_{1}\right)}{\sqrt{a\left(y_{1}\right)}}-\ln \frac{w\left(y_{1}, z_{1}\right)}{\sqrt{a\left(z_{1}\right)}}\right]\right\} .
$$

Since $w\left(y_{1}, y_{1}\right)=\boldsymbol{a}\left(y_{1}\right)$ this gives

$$
\tau(y, z)=\exp \left\{\frac{1}{2} \ln \frac{\sqrt{a\left(z_{1}\right) a\left(y_{1}\right)}}{w\left(z_{1}, y_{1}\right)}\right\}=\frac{\sqrt[4]{a\left(z_{1}\right) a\left(y_{1}\right)}}{\sqrt{w\left(y_{1}, z_{1}\right)}} .
$$

We now determine the parallel displacement of the canonical basis vectors $\left(\partial_{1}(y), \ldots, \partial_{n}(y)\right)$ along geodesics starting from a fixed point $y \in M$. 
Proposition 9 Let $y$ and $z$ be two points in $M$ with $y_{1} \neq z_{1}$. Let us denote by $\mathfrak{b} \in \mathbb{R}^{n-2}$ the vector

$$
\mathfrak{b}:=\frac{1}{z_{1}-y_{1}}(\tilde{z}-\tilde{y}) W\left(y_{1}, z_{1}\right)
$$

and by $P=P\left(x_{1}\right)$ the matrix given by the initial value problem

$$
\dot{P}=-\frac{1}{2} P \dot{A} A^{-1} \quad P\left(y_{1}\right)=E .
$$

Then the parallel displacement $\mathfrak{p}: T_{y} M \longrightarrow T_{z} M$ along the geodesic joining $y$ with $z$ satisfies

$$
\begin{aligned}
\mathfrak{p}\left(\partial_{1}(y)\right)= & \partial_{1}(z)+\frac{1}{2} \mathfrak{b}\left[A^{-1}\left(y_{1}\right)\left(2 P\left(z_{1}\right)-E\right)-A^{-1}\left(z_{1}\right)\right] \mathfrak{b}^{t} \partial_{2}(z) \\
& +\left(\mathfrak{b}\left[A^{-1}\left(z_{1}\right)-A^{-1}\left(y_{1}\right) P\left(z_{1}\right)\right]\right)_{K} \partial_{K}(z) \\
\mathfrak{p}\left(\partial_{2}(y)\right)= & \partial_{2}(z) \\
\mathfrak{p}\left(\partial_{L}(y)\right)= & P_{L K}\left(z_{1}\right) \partial_{K}(z)+\left(\left(E-P\left(z_{1}\right)\right) \mathfrak{b}^{t}\right)_{L} \partial_{2}(z) \quad L=3, \ldots, n
\end{aligned}
$$

Proof: Let $\gamma$ be the geodesic joining $y$ with $z$ and let $X(t)=a_{j}(t) \partial_{j}(\gamma(t))$ be a vector field parallel along $\gamma$. Denote $\mathcal{A}(t):=A\left(\gamma_{1}(t)\right)$ and $W=W\left(y_{1}, z_{1}\right)$. If we insert the results of Lemma 1 and Proposition 5 into the equation

$$
a_{i}^{\prime}(t)+\sum_{j, k} \gamma_{k}^{\prime}(t) a_{j}(t) \Gamma_{k j}^{i}(t)=0 \quad i=1, \ldots n
$$

for the parallel displacement, we obtain

$$
\begin{aligned}
a_{1}^{\prime}(t) & =0 \\
a_{2}^{\prime}(t) & =\frac{1}{2} \mathfrak{b}\left(\mathcal{A}^{-1} \mathcal{A}^{\prime}\right)(t) \tilde{a}^{t}(t) \\
\tilde{a}^{\prime t}(t) & =-\frac{1}{2}\left\{\left(\mathcal{A}^{-1} \mathcal{A}^{\prime} \tilde{a}^{t}\right)(t)-a_{1}(t)\left(\mathcal{A}^{-1}\right)^{\prime}(t) \mathfrak{b}^{t}\right\}
\end{aligned}
$$

where $\widetilde{a}(t)=\left(a_{3}(t), \ldots, a_{n}(t)\right)$. Since $\partial_{2}=T$ is the isotropic parallel vector field on the standard plane wave manifold, (18) holds.

Now, let $X(t)=a_{L j}(t) \partial_{j}(\gamma(t))$ be the parallel displacement of $\partial_{L}(y)$. Then the initial condition yields $a_{L 1}(t)=0$. Therefore

$$
\tilde{a}_{L}^{\prime t}(t)=-\frac{1}{2} \mathcal{A}^{-1} \mathcal{A}^{\prime} \tilde{a}_{L}^{t}(t), \quad a_{L K}(0)=\delta_{L K}
$$

This is solved by $a_{L K}(t):=P_{L K}\left(\gamma_{1}(t)\right)$. For $a_{L 2}(t)$ it follows $a_{L 2}^{\prime}(t)=-\left\langle\mathfrak{b}, \tilde{a}_{L}^{\prime}(t)\right\rangle$ and the initial condition gives $a_{L 2}(t)=\mathfrak{b}_{L}-\left\langle\mathfrak{b}, \tilde{a}_{L}(t)\right\rangle$. This proves $(19)$.

Now, let $X(t)=a_{1 j}(t) \partial_{j}(\gamma(t))$ be the parallel displacement of $\partial_{1}(y)$. Then $a_{11}(t)=1$. Therefore

$$
\tilde{a}_{1}^{\prime t}(t)=-\frac{1}{2} \mathcal{A}^{-1} \mathcal{A}^{\prime} \tilde{a}_{1}^{t}(t)+\frac{1}{2}\left(\mathcal{A}^{-1}\right)^{\prime} \mathfrak{b}^{t}, \quad \tilde{a}_{1}(0)=0 .
$$


This initial value problem is solved by the vector

$$
\tilde{a}_{1}(t)=\mathfrak{b}\left[\mathcal{A}^{-1}(t)-A^{-1}\left(y_{1}\right) P\left(\gamma_{1}(t)\right)\right] .
$$

For $a_{12}$ it follows

$$
a_{12}^{\prime}(t)=-\tilde{a}_{1}^{\prime}(t) \cdot \mathfrak{b}^{t}+\frac{1}{2} \mathfrak{b}\left(\mathcal{A}^{-1}\right)^{\prime}(t) \mathfrak{b}^{t}
$$

and the initial condition gives

$$
a_{12}(t)=\frac{1}{2} \mathfrak{b}\left[-\mathcal{A}^{-1}(t)+A^{-1}\left(y_{1}\right)\left(2 P\left(\gamma_{1}(t)\right)-E\right)\right] \mathfrak{b}^{t} .
$$

This proves (17).

\subsection{The Dirac operator of a standard plane wave manifold}

In this section $(M, g)$ denotes a standard plane wave manifold as it was defined in Chapter 4.1. Since $M$ is parallelizable, $(M, g)$ is an oriented Lorentzian manifold with a unique (trivial) spinor structure $Q$. By $S$ we denote the associated spinor bundle $S=Q \times \times_{\operatorname{Spin}(n, 1)} \Delta_{n, 1}$.

Let $y$ be a fixed point in $M$. We choose a $(n-2) \times(n-2)-$ matrix $C$ such that $C A\left(y_{1}\right) C^{t}=E$. Then

$$
\begin{aligned}
& s_{1}(y):=\frac{1}{\sqrt{2}}\left(\partial_{1}(y)-\partial_{2}(y)\right) \\
& s_{2}(y):=\frac{1}{\sqrt{2}}\left(\partial_{1}(y)+\partial_{2}(y)\right) \\
& s_{I}(y):=C_{I J} \partial_{J}(y), \quad I=3, \ldots, n
\end{aligned}
$$

is an orthonormal basis in $T_{y} M$. We denote by $s=\left(s_{1}, \ldots, s_{n}\right)$ the global orthonormal basis on $M$ arising from $\left(s_{1}(y), \ldots, s_{n}(y)\right)$ by parallel displacement along geodesics. Let the orientation of $M$ be fixed by this basis $s$. $\hat{s}$ denotes a lift of $s$ in the spinor structure $Q$.

Proposition 10 Let $v_{1}, \ldots, v_{N}$ be a basis in the spinor modul $\Delta_{n, 1}$ and let us denote by $\eta_{1}, \ldots, \eta_{N} \in \Gamma(S)$ the basis sections in the spinor bundle defined by

$$
\eta_{\varepsilon}(x):=\left[\hat{s}(x), v_{\varepsilon}\right], \quad \varepsilon=1, \ldots, N=2^{\left[\frac{n}{2}\right]} .
$$

Then over the open submanifold $\left\{x \in M \mid x_{1} \neq y_{1}\right\}$ the spinor derivative of $\eta_{\varepsilon}$ is given by

$$
\begin{aligned}
\nabla_{\partial_{1}}^{S} \eta_{\varepsilon} & =\frac{1}{\sqrt{2}} \sum_{I} \omega_{1 I}\left(\partial_{1}\right) T \cdot s_{I} \cdot \eta_{\varepsilon}+\frac{1}{2} \sum_{I<J} \omega_{I J}\left(\partial_{1}\right) s_{I} \cdot s_{J} \cdot \eta_{\varepsilon} \\
\nabla_{\partial_{2}}^{S} \eta_{\varepsilon} & =0 \\
\nabla_{\partial_{K}}^{S} \eta_{\varepsilon} & =\frac{1}{\sqrt{2}} \sum_{I} \omega_{1 I}\left(\partial_{K}\right) T \cdot s_{I} \cdot \eta_{\varepsilon} \quad K=3, \ldots, n
\end{aligned}
$$


where $\omega_{i j}=g\left(\nabla s_{i}, s_{j}\right)$ are the connection forms of the Levi-Civita connection with respect to the basis $\left(s_{1}, \ldots, s_{n}\right)$. The connection coefficients $\omega_{1 I}\left(\partial_{K}\right)$ and $\omega_{I J}\left(\partial_{1}\right)$ depend only on the first variable $x_{1}$.

Proof: The spinor derivative of $\eta_{\varepsilon}$ is given by

$$
\nabla_{X}^{S} \eta_{\varepsilon}=\frac{1}{2} \sum_{i<j} \varepsilon_{i} \varepsilon_{j} \omega_{i j}(X) s_{i} \cdot s_{j} \cdot \eta_{\varepsilon}
$$

Hence we have to calculate $\omega_{i j}$.

Using Proposition 9 we obtain over $\left\{x \in M \mid x_{1} \neq y_{1}\right\}$

$$
\begin{aligned}
s_{1}= & \frac{1}{\sqrt{2}}\left[\partial_{1}+\left(\frac{1}{2} \mathfrak{b}\left[A^{-1}\left(y_{1}\right)(2 P-E)-A^{-1}\right] \mathfrak{b}^{t}-1\right) \partial_{2}\right. \\
& \left.+\left(\mathfrak{b}\left[A^{-1}-A^{-1}\left(y_{1}\right) P\right]\right)_{K} \partial_{K}\right] \\
s_{2}= & \frac{1}{\sqrt{2}}\left[\partial_{1}+\left(\frac{1}{2} \mathfrak{b}\left[A^{-1}\left(y_{1}\right)(2 P-E)-A^{-1}\right] \mathfrak{b}^{t}+1\right) \partial_{2}\right. \\
& \left.+\left(\mathfrak{b}\left[A^{-1}-A^{-1}\left(y_{1}\right) P\right]\right)_{K} \partial_{K}\right] \\
s_{I}= & (C P)_{I K} \partial_{K}+\left(C(E-P) \mathfrak{b}^{t}\right)_{I} \partial_{2}
\end{aligned}
$$

where $\mathfrak{b}(x)$ is the vector

$$
\mathfrak{b}(x)=\frac{1}{x_{1}-y_{1}}(\tilde{x}-\tilde{y}) W\left(y_{1}, x_{1}\right)
$$

and $P=P\left(x_{1}\right)$ is the matrix given by

$$
\dot{P}=-\frac{1}{2} P \dot{A} A^{-1} \quad P\left(y_{1}\right)=E .
$$

Since by Lemma $1 \Gamma_{2 i}^{j}=0$ for all $i, j=1, \ldots, n$ and the coefficients of $s_{i}$ with respect to $\left(\partial_{1}, \ldots, \partial_{n}\right)$ do not depend on the second variable, it follows

$$
\omega_{i j}\left(\partial_{2}\right)=0 \quad i, j=1, \ldots, n .
$$

This proves (21). Because of $s_{2}=\sqrt{2} \partial_{2}+s_{1}$ and $\Gamma_{i j}^{1}=0$ it is

$$
\omega_{12}\left(\partial_{k}\right)=\sqrt{2} g\left(\nabla_{\partial_{k}} s_{1}, \partial_{2}\right)=0
$$

Furthermore, since $T=\partial_{2}$ is parallel, one obtains

$$
\omega_{2 I}-\omega_{1 I}=g\left(\nabla\left(s_{2}-s_{1}\right), s_{I}\right)=\sqrt{2} g\left(\nabla \partial_{2}, s_{I}\right)=0 .
$$

Inserting (28) and (29) in (23) gives

$$
\nabla_{X}^{S} \eta_{\varepsilon}=\frac{1}{\sqrt{2}} \sum_{I} \omega_{1 I}(X) T \cdot s_{I} \cdot \eta_{\varepsilon}+\frac{1}{2} \sum_{I<J} \omega_{I J}(X) s_{I} \cdot s_{J} \cdot \eta_{\varepsilon}
$$


Now, a direct calculation using Lemma 1 and (24), (25), (26) shows, that

$$
\begin{aligned}
\omega_{1 I}\left(\partial_{1}\right) & =\frac{1}{\sqrt{2}}\left[\left(C P A \dot{R}^{t}\right)_{I}+\frac{1}{2}\left(C P \dot{A} R^{t}\right)_{I}\right] \\
\omega_{I J}\left(\partial_{1}\right) & =\left(C \dot{P} A P^{t} C^{t}+\frac{1}{2} C P \dot{A} P^{t} C^{t}\right)_{I J} \\
\omega_{1 I}\left(\partial_{K}\right) & =\frac{1}{\sqrt{2}}\left[\frac{1}{2}(C P \dot{A})_{I K}+\frac{1}{x_{1}-y_{1}}\left(C P\left[E-A P^{t} A^{-1}\left(y_{1}\right)\right] W\right)_{I K}\right] \\
\omega_{I J}\left(\partial_{K}\right) & =0
\end{aligned}
$$

where $R=R(x)$ is the vector $R=\mathfrak{b}\left[A^{-1}-A^{-1}\left(y_{1}\right) P\right]$ and $\dot{R}$ denotes the derivative with respect to $x_{1}$. Hence with (30) this proves (20) and (22). The connection coefficients $\omega_{I J}\left(\partial_{1}\right)$ and $\omega_{1 I}\left(\partial_{K}\right)$ depend only on the first variable $x_{1}$.

Proposition 11 On the open submanifold $\left\{x \in M \mid x_{1} \neq y_{1}\right\}$ the Dirac operator satisfies

$$
D\left(\eta_{\varepsilon}\right)=\sum_{I<J} f_{I J} T \cdot s_{I} \cdot s_{J} \cdot \eta_{\varepsilon}+f T \cdot \eta_{\varepsilon}
$$

where $f_{I J}$ and $f$ are functions depending only on $x_{1}$.

Proof: Since $\nabla_{\partial_{2}}^{S} \eta_{\varepsilon}=0$ it is $\nabla_{s_{1}}^{S} \eta_{\varepsilon}=\nabla_{s_{2}}^{S} \eta_{\varepsilon}$. Hence

$$
\begin{aligned}
D\left(\eta_{\varepsilon}\right) & =\sum_{i} \varepsilon_{i} s_{i} \cdot \nabla_{s_{i}}^{S} \eta_{\varepsilon} \\
& =\left(s_{2}-s_{1}\right) \cdot \nabla_{s_{1}}^{S} \eta_{\varepsilon}+\sum_{I} s_{I} \cdot \nabla_{s_{I}}^{S} \eta_{\varepsilon} \\
& =T \cdot\left(\nabla_{\partial_{1}} \eta_{\varepsilon}+\left(\mathfrak{b}\left[A^{-1}-A^{-1}\left(y_{1}\right) P\right]\right)_{K} \nabla_{\partial_{K}}^{S} \eta_{\varepsilon}\right)+\sum_{I K}(C P)_{I K} s_{I} \cdot \nabla_{\partial_{K}}^{S} \eta_{\varepsilon} .
\end{aligned}
$$

Using $T \cdot T=-g(T, T)=0$ for the isotropic vector field $T$ Proposition 10 gives

$$
D\left(\eta_{\varepsilon}\right)=\frac{1}{2} \sum_{I<J} \omega_{I J}\left(\partial_{1}\right) T \cdot s_{I} \cdot s_{J} \cdot \eta_{\varepsilon}-\frac{1}{\sqrt{2}} \sum_{I J K}(C P)_{I K} \omega_{1 J}\left(\partial_{K}\right) T \cdot s_{I} \cdot s_{J} \cdot \eta_{\varepsilon} .
$$

Since $P, \omega_{I J}\left(\partial_{1}\right)$ and $\omega_{1 J}\left(\partial_{K}\right)$ depend only on $x_{1}$, this proves the proposition.

Proposition 12 Let $h \in C^{\infty}(M)$ be a function depending only on $x_{1}$. Then

$$
D^{2}\left(h \eta_{\varepsilon}\right)=0, \quad \varepsilon=1, \ldots, N .
$$


Proof: Since all sections and functions are continuous it is enough to prove the assertion on the open submanifold $\left\{x \in M \mid x_{1} \neq y_{1}\right\}$.

For the Dirac operator of a Lorentzian spin manifold the following commutation rules are valid

$$
\begin{aligned}
D(f \varphi) & =f D \varphi+\operatorname{grad} f \cdot \varphi \\
D(X \cdot \varphi) & =-X \cdot D \varphi-2 \nabla_{X}^{S} \varphi+\sum_{i} \varepsilon_{i} s_{i} \cdot \nabla_{s_{i}} X \cdot \varphi
\end{aligned}
$$

where $f$ is a function, $X$ is a vector field and $\varphi$ is a spinor field. If $h$ is a function on the standard plane wave manifold depending only on $x_{1}$ then $\operatorname{grad} h=\dot{h} T$. Hence from (31) it follows

$$
D\left(h \eta_{\varepsilon}\right)=\dot{h} T \cdot \eta_{\varepsilon}+h D \eta_{\varepsilon} .
$$

Since $s_{I} \cdot s_{J} \cdot \eta_{\varepsilon}=A[\mathrm{IJ}]_{\varepsilon \delta} \eta_{\delta}$, where $A[\mathrm{IJ}]_{\varepsilon \delta}$ are constant functions given by the matrix representation of the linear map $e_{I} \cdot e_{J} \cdot$ on the spinor modul $\Delta_{n, 1}$ with respect to the basis $\left(v_{1}, \ldots, v_{N}\right)$, Proposition 11 results that $D\left(h \eta_{\varepsilon}\right)$ can be expressed in the form

$$
D\left(h \eta_{\varepsilon}\right)=\tilde{h}_{\varepsilon \delta} T \cdot \eta_{\delta}
$$

where $\tilde{h}_{\varepsilon \delta}$ are functions depending only on $x_{1}$. Hence

$$
D^{2}\left(h \eta_{\varepsilon}\right)=\dot{\tilde{h}}_{\varepsilon \delta} T \cdot T \cdot \eta_{\delta}+\tilde{h}_{\varepsilon \delta} D\left(T \cdot \eta_{\delta}\right)
$$

Since $T$ is parallel and $\nabla_{T}^{S} \eta_{\varepsilon}=0$, from (32) it follows $D(T \cdot \varphi)=-T \cdot D \varphi$. Using $T \cdot T=-g(T, T)=0$ this gives under consideration of Proposition 11

$$
D^{2}\left(h \eta_{\varepsilon}\right)=-\tilde{h}_{\varepsilon \delta} T \cdot D \eta_{\delta}=0 .
$$

Theorem 9 The Hadamard coefficients $U_{k} \in \Gamma\left(M \times M, S^{*} \otimes S\right)$ of the square of the Dirac operator on a standard plane wave manifold vanish for all $k \geq 1$.

Proof: From Proposition 3 it is known that the Hadamard coefficient $U_{0}$ satisfies

$$
U_{0}(y, z)=\frac{1}{\tau(y, z)} \mathcal{P}(y, z)
$$

where $\tau$ is the function described in Proposition 8 and $\mathcal{P}(y, z)$ is the parallel displacement from $T_{y} M$ to $T_{z} M$ along the geodesic joining $y$ with $z$.

Since the basis $\left(s_{1}, \ldots, s_{n}\right)$ is parallel along geodesics starting from $y$, the sections $\eta_{\varepsilon}=\left[\hat{s}, v_{\varepsilon}\right]$ in the spinor bundle are also parallel along these geodesics. Therefore the parallel displacement $\mathcal{P}(y, z)$ can be expressed by

$$
\mathcal{P}(y, z)=\sum_{\varepsilon} \eta_{\varepsilon}^{*}(y) \otimes \eta_{\varepsilon}(z) .
$$


According to Proposition 8 the function $\tau(y, \cdot)$ depends only on the first variable. Using Proposition 12 we obtain

$$
D^{2}\left(U_{0}(y, \cdot)\right)=0 .
$$

Then Proposition 3 shows that $U_{1}=0$. Hence all Hadamard coefficients $U_{k}$ for $k \geq 1$ vanish.

To be of Huygens type is a local property of an operator. Therefore we obtain under consideration of Proposition 8 from the Hadamard criterion

Theorem 10 Let $\left(M^{n}, g\right)$ be a plane wave spin manifold of even dimension $n \geq 4$. Then the square of the Dirac operator is of Huygens type.

\section{The Huygens property for twisted Dirac operators on 4-dimensional Lorentzian spin manifolds}

In this chapter we consider the Huygens property for twisted Dirac operators. Let $\left(M^{n}, g\right)$ be a Lorentzian spin manifold with spinor bundle $S$. Furthermore, let $P$ be a $G$-principal bundle over $M, \rho: G \longrightarrow G L(V)$ a complex representation of $G$ and $E=P \times_{\rho} V$ the associated complex vector bundle. Each connection $A$ of $P$ induces a Dirac operator

$$
D_{A}: \Gamma(S \otimes E) \longrightarrow \Gamma(S \otimes E)
$$

with values in $E$ defined by

$$
D_{A}=D \otimes 1+\sum_{i=1}^{n} \varepsilon_{i} s_{i} \cdot \otimes \nabla_{s_{i}}^{A},
$$

where $D$ is the Dirac operator, $\nabla^{A}$ is the covariant derivative on $E$ given by $A, \cdot$ is the Clifford multiplication and $\left(s_{1}, \ldots, s_{n}\right)$ is a local orthonormal basis on $(M, g)$. The Weitzenböck formula for $D_{A}^{2}$ is given by

$$
D_{A}^{2}=\Delta^{\nabla}+\frac{1}{4} R+Q_{A},
$$

where $R$ is the scalar curvature of $\left(M^{n}, g\right)$,

$$
\begin{aligned}
\nabla & =\nabla^{S} \otimes 1+1 \otimes \nabla^{A} & & \text { and } \\
Q_{A} & =\sum_{i<j} \varepsilon_{i} \varepsilon_{j} s_{i} \cdot s_{j} \cdot \otimes F^{A}\left(s_{i}, s_{j}\right), & & F^{A} \text { curvature of } \nabla^{A}
\end{aligned}
$$

([LM89], p.164). In even dimension $n$ the spinor bundle $S$ splits into the sum $S=S^{+} \oplus$ $S^{-}$of positive and negative spinors. Let us denote by $D_{A}^{ \pm}: \Gamma\left(S^{ \pm} \otimes E\right) \longrightarrow \Gamma\left(S^{\mp} \otimes E\right)$ the restrictions of $D_{A}$ to the corresponding subbundles. Then

$$
D_{A}^{2}=\left(\begin{array}{cc}
D_{A}^{-} D_{A}^{+} & 0 \\
0 & D_{A}^{+} D_{A}^{-}
\end{array}\right): \Gamma\left(\begin{array}{c}
S^{+} \otimes E \\
S^{-} \otimes E
\end{array}\right) \rightarrow \Gamma\left(\begin{array}{c}
S^{+} \otimes E \\
S^{-} \otimes E
\end{array}\right) .
$$


We first proof the following proposition

Proposition 13 Let $\left(M^{n}, g\right)$ be a space and time oriented Lorentzian spin manifold of even dimension $n=2 m \geq 4$. Then the operator $D_{A}^{-} D_{A}^{+}: \Gamma\left(S^{+} \otimes E\right) \longrightarrow \Gamma\left(S^{+} \otimes E\right)$ is of Huygens type if and only if the operator $D_{A}^{+} D_{A}^{-}: \Gamma\left(S^{-} \otimes E\right) \longrightarrow \Gamma\left(S^{-} \otimes E\right)$ is of Huygens type.

Proof: In [Bau94] it was proved that on the spinor bundle $S=S^{+} \oplus S^{-}$of an even dimensional space and time oriented Lorentzian spin manifold there exists an antiunitary map $C: S^{ \pm} \longrightarrow S^{\mp}$ such that

$$
\begin{aligned}
D C & =-C D & & \\
X C & =-C X & & \text { for all vector fields } X \\
C^{2} & =(-1)^{\alpha(n)} I d & & \text { where } \alpha(n)=(m(m+1) / 2)+1 .
\end{aligned}
$$

If we extend $C$ to $S \otimes E$ by $C(\phi \otimes e)=C \phi \otimes e$, from (35), (36) and (37) it follows

$$
D_{A}^{+} D_{A}^{-}=(-1)^{\alpha(n)} C^{+} D_{A}^{-} D_{A}^{+} C^{-} .
$$

Now, let $\Omega_{0}$ be a causal domain and $x \in \Omega_{0}$. Let $G_{+}(x)$ and $G_{-}(x)$ be the forward and backward fundamental solutions of $D_{A}^{+} D_{A}^{-}$with respect to $\left(\Omega_{0}, x\right)$. We denote by $H_{ \pm}(x) \in \mathcal{D}_{ \pm}^{\prime}\left(\Omega_{0},\left(S^{+} \otimes E\right)^{*} ;\left(S^{+} \otimes E\right)_{x}^{*}\right)$ the following distribution

$$
\left\langle H_{ \pm}(x), \psi\right\rangle:=(-1)^{\alpha(n)}\left(C^{+}\right)_{x}^{*}\left\langle G_{ \pm}(x),\left(C^{-}\right)^{*} \psi\right\rangle \quad \psi \in \Gamma_{0}\left(\left(S^{+} \otimes E\right)^{*}\right)
$$

where $\left(C^{ \pm}\right)^{*}$ denotes the maps induced by $C^{ \pm}$on the dual bundles. Using (37) and (38) it is easy to check, that $H_{ \pm}(x)$ is the fundamental solution of $D_{A}^{-} D_{A}^{+}$with respect to $\left(\Omega_{0}, x\right)$ and that the support of $H_{ \pm}(x)$ coincides with that of $G_{ \pm}(x)$. Hence the operator $D_{A}^{+} D_{A}^{-}$is Huygens if and only if $D_{A}^{-} D_{A}^{+}$is Huygens.

Now, we cosider the Huygens property for the square $D_{A}^{2}$ of the twisted Dirac operator on a 4 -dimensional Lorentzian spin manifold. In case of $U(1)$-connections the following statement was proved by Illge ([IIl88]).

Theorem 11 Let $\left(M^{4}, g\right)$ be a space and time oriented analytic Lorentzian spin manifold of dimension 4, let $f$ be a smooth function on $M$ and $D_{A}: \Gamma(S \otimes E) \longrightarrow \Gamma(S \otimes E)$ be the Dirac operator coupled to a connection $A$ of a complex vector bundle $E$. Let us denote by $P(A)$ one of the operators $D_{A}^{2}, D_{A}^{+} D_{A}^{-}$or $D_{A}^{-} D_{A}^{+}$. If $P(A)-f$ is of Huygens type, then the scalar curvature $R$ of $(M, g)$ is constant and equals $12 f$.

If $R$ is constant and non zero, then $P(A)-\frac{1}{12} R$ is of Huygens type if and only if the connection $A$ is flat and $\left(M^{4}, g\right)$ is of constant sectional curvature.

If $R=0$, then $P(A)$ is of Huygens type if and only if $A$ is flat and $\left(M^{4}, g\right)$ is conformally flat or locally isometric to a plane wave manifold. 
Proof: According to Proposition 13 it is enough to consider the case $P(A)=D_{A}^{2}$. Suppose that the operator $P(A)-f$ is of Huygens type. According to Theorem 4 this implies that its Cotton invariant $C$ vanishes. From the Weitzenböck formula (34) of $D_{A}^{2}$ we obtain for the Cotton invariant $C=\frac{1}{12} R-f+Q_{A}$. Hence we have the condition

$$
Q_{A}=\sum_{i<j} \varepsilon_{i} \varepsilon_{j} s_{i} \cdot s_{j} \cdot \otimes F^{A}\left(s_{i}, s_{j}\right)=\left(f-\frac{1}{12} R\right) I d_{S \otimes E} .
$$

For the calculations we identify the complexified Clifford algebra $\mathrm{Cliff}_{4,1}^{\mathbb{C}}$ of the Minkowski space with the algebra of complex $4 \times 4$-matrices using the map $\Phi$ given by

$$
\Phi\left(e_{1}\right)=i E \otimes U \quad \Phi\left(e_{2}\right)=E \otimes V \quad \Phi\left(e_{3}\right)=U \otimes T \quad \Phi\left(e_{4}\right)=V \otimes T,
$$

where $\left(e_{1}, \ldots, e_{4}\right)$ denotes the canonical basis of the Minkowski space and

$$
U=\left(\begin{array}{cc}
i & 0 \\
0 & -i
\end{array}\right) \quad V=\left(\begin{array}{cc}
0 & i \\
i & 0
\end{array}\right) \quad E=\left(\begin{array}{cc}
1 & 0 \\
0 & 1
\end{array}\right) \quad T=\left(\begin{array}{cc}
0 & -i \\
i & 0
\end{array}\right) .
$$

Let $u(\varepsilon):=\left(\begin{array}{c}1 \\ -\varepsilon i\end{array}\right), \varepsilon= \pm 1$, and $u\left(\varepsilon_{1}, \varepsilon_{2}\right):=u\left(\varepsilon_{1}\right) \otimes u\left(\varepsilon_{2}\right)$. Let us denote by $\tilde{s}$ a lift of the local orthonormal frame $s=\left(s_{1}, \ldots, s_{4}\right)$ into the spinor structure and $\eta\left(\varepsilon_{1}, \varepsilon_{2}\right):=\left[\tilde{s}, u\left(\varepsilon_{1}, \varepsilon_{2}\right)\right]$ the corresponding local sections in the spinor bundle $S$. Then $(\eta(1,1), \eta(-1,-1))$ is a local basis in $S^{+}$and $(\eta(1,-1), \eta(-1,1))$ is a local basis in $S^{-}$. If we use this basis the homomorphism $Q_{A}^{ \pm}$is given by

$$
Q_{A}^{ \pm}\left(\begin{array}{c}
\psi_{1} \\
\psi_{2}
\end{array}\right)=\left(\begin{array}{c}
\left( \pm F_{12}+i F_{34}\right) \psi_{1}+\left( \pm i F_{13} \mp F_{14}-i F_{23}+F_{24}\right) \psi_{2} \\
\left(\mp i F_{13}+\mp F_{14}-i F_{23}-F_{24}\right) \psi_{1}+\left(\mp F_{12}-i F_{34}\right) \psi_{2}
\end{array}\right),
$$

where $F_{i j}=F^{A}\left(s_{i}, s_{j}\right)$. This shows that $Q_{A}^{+}$is a multiple of the identity if and only if $F^{A}=i * F^{A}$ and $Q_{A}^{-}$is a multiple of the identity if and only if $F^{A}=-i * F^{A}$. Here $*$ is the Hodge operator of the Lorentzian metric. Hence condition (39) implies that $A$ is flat: $F^{A}=0$. Since the Huygens property is a local one we can assume that $M$ is simply connected. Then, because of the flatness of $A$, there exists a global trivialization of $E$ by $\nabla^{A}$ - parallel sections. Using this trivialization the bundle $S \otimes E$ can be identified with the sum $(S \oplus \ldots \oplus S)$ of the spinor bundle such that the Dirac operator $D_{A}$ acts on each factor $S$ as the uncoupled Dirac operator $D$ (see (33)). Hence $P(A)-f$ is Huygens if and only if $A$ is flat and the uncoupled shifted Dirac operator $D^{2}-f$ is Huygens. Then the assertion follows from the Theorem of Wünsch (Theorem 1).

\section{References}

[Bau81] H. Baum. Spin-Strukturen und Dirac-Operatoren über pseudo-Riemannschen Mannigfaltigkeiten, volume 41 of Teubner-Texte zur Mathematik. Teubner-Verlag, Leipzig, 1981. 
[Bau94] H. Baum. A remark on the spectrum of the Dirac operator on pseudoRiemannian spin manifolds. SFB 288-Preprint No. 136, 1994.

[BK96] H. Baum and I. Kath. Normally hyperbolic operators, the Huygens property and conformal geometry. SFB 288 Preprint No. 212, 1996.

[BV94] Y.Y. Berest and A.P. Veselov. Huygens' principle and integrability (russ.) Uspechi mat. Nauk, 49(6):7-78, 1994.

[CGLS86] M. Cahen, S. Gutt, L. Lemaire, and P. Spindel. Killing spinors. Bull. Soc. Math. Belg., 38:76-102, 1986.

[Fri75] F.G. Friedlander. The Wave Equation on a curved Space Time. Camb. Univ. Press, 1975.

[Gün65] P. Günther. Ein Beispiel einer nichttrivialen Huygensschen Differentialgleichung mit vier unabhängigen Veränderlichen. Archive Rat. Mech. and Analysis, 18:103-106, 1965.

[Gün88] P. Günther. Huygens' Principle and Hyperbolic Equations, volume 5 of Persp. in Math. Acad. Press Inc., Boston, 1988.

[Gün91] P. Günther. Huygens' principle and Hadamard's conjecture. Mathematical Intelligencer, 13:56-63, 1991.

[Had23] J. Hadamard. Lectures on Cauchy's Problem in Linear Partial Differential Equations. Yale Univ. Press, New Haven, 1923.

[Hel94] S. Helgason. Geometric Analysis on Symmetric Spaces, volume 39 of Mathematical Serveys and Monographs. AMS, Providence, Rhode Island, 1994.

[Ill88] R. Illge. On Huygens' principle for the relativistic higher spin wave equation of Buchdahl and Wünsch in presence of a gravitational and electromagnetic field. Math. Nachr., 139:237-243, 1988.

[LM89] H.B. Lawson and M-L. Michelsohn. Spin Geometry. Princton Univ. Press, 1989.

[Sch71] R. Schimming. Zur Gültigkeit des Huygensschen Prinzips bei einer speziellen Metrik. ZAMM, 51:201-208, 1971.

[Sch74] R. Schimming. Riemannsche Räume mit ebenfrontiger und ebener Symmetrie. Mathematische Nachrichten, 59:129-162, 1974.

[SS94] R. Schimming and H. Schlichtkrull. Helmholtz operators on harmonic manifolds. Acta Math, 173(2):235-258, 1994.

[Wün78] V. Wünsch. Cauchy-Problem und Huygenssches Prinzip bei einigen Klassen spinorieller Feldgleichungen I. Beiträge zur Analysis, 12:47-76, 1978. 
[Wün79] V. Wünsch. Cauchy-Problem und Huygenssches Prinzip bei einigen Klassen spinorieller Feldgleichungen II. Beiträge zur Analysis, 13:147-177, 1979.

[Wün80] V. Wünsch. Selbstadjungierte Huygenssche Differentialgleichungen 2. Ordnung für nicht-skalare Spintensorfelder. Math. Nachr., 94:211-242, 1980.

[Wün94] V. Wünsch. Moments and Huygens' principle for conformally invariant field equations in curved space-times. Ann. Henri Poincare Vol., 60(4):433-455, 1994.

\author{
Helga Baum \\ Institut für Reine Mathematik \\ Humboldt-Universität zu Berlin \\ Sitz: Ziegelstr. 13a \\ 10099 Berlin \\ Germany \\ email: baum@mathematik.hu-berlin.de
}


Sfb 288 Preprints are availlable at:

http: //www-sfb288. math.tu-berlin. de

List of most recent 50 sfb288 preprints

166 V. Bazhanov, A. Bobenko, N. Reshetikhin: Quantum Discrete Sine-Gordon Model at Roots of 1: Integrable quantum System on the Integrable Classical Background

167 U. Bunke, M. Olbrich: Fuchsian groups of the second kind and representations carried by the limit set

168 H. Ferguson, A. Gray, St. Markvorsen: Costa's Minimal Surface via Mathematica

169 J. Dorfmeister, I. McIntosh, F. Pedit, H. Wu: On the Meromorphic Potential for a Harmonic Surface in a k-Symmetric space

170 M. Lüdde: Notes on generalised Magnus modules over the braid group

171 V. Bach, J. Fröhlich, I. M. Sigal: Mathematical Theory of NonRelativistic Matter and Radiation

172 P. Contucci, A. Knauf: The Phase Transition of the Number-Theoretical Spin Chain

173 K. Mohnke: On Seiberg-witten Equations on Symplectic 4-manifolds

174 D. Ferus, F. Pedit: Curved Flats in Symmetric Spaces

175 M. Hinze: on the Numerical Approximation and Computation of MinimalSurface-Continua bounded by one-Parameter-Families of Polygonal Contours

$176 \mathrm{U}$. Hertrich-Jeromin: On Conformally flat hypersurfaces, Curved flats and Cyclic sytems

177 U. Bunke, M. Olbrich: Cohomological properties of the smooth globalization of a Harish-Chandra module

178 F. Nill, K. Szlachányi: Quantum Chains of Hopf Algebras with Quantum Double Cosymmetry

179 St. Meißner, B.-D. Dörfel: Ground state and low excitations of an integrable chain with alternating spins

180 Ch. Bär: Harmonic Spinors for Twisted Dirac Operators

181 F. Constantinescu, M. Luedde: The Alexander and Jones-invariants and the Burau module

182 A. Bobenko, U. Eitner, A. Kitaev: Harmonic Inverse Mean Curvature Surfaces and Painleve Equations

183 K.-D. Kirchberg: Kählerian E-spinors

$184 \mathrm{~J}$. Kellendonk: Integer Groups of Coinvariants Associated to Octagonal Tilings

185 V. Kostrykin, R. Schrader: Ionization of Atoms and Molecules by Short Strong Laser Pulses

186 M. J. Pflaum: A new concept of deformation quantization I. Normal order quantization on cotangent bundles

187 J. Brüning: The local index theorem without smoothness

188 U. Bunke, M. Olbrich: Cohomological properties of the canonical globalizations of Harish-Chandra modules

189 U. Bunke, M. Olbrich: Group cohomology and the singularities of the Selberg zeta function associated to a Kleinian group 
190 H. Gollek: Deformations of isotropic curves in C3 and minimal surfaces in R3

191 F. Guerra, A. Knauf: Free Energy and Correlations of the NumberTheoretical Spin-Chain

192 H.-W. Wiesbrock: Symmetries and Modular Intersections of von-NeumannAlgebras

193 H.-W. Wiesbrock: Modular Intersections of von-Neumann-Algebras in Quantum Field Theory

194 H. Karcher, K. Polthier: Construction of Triply Periodic Minimal Surfaces

195 V. Bach, J. Poelchau: Accuracy of the Hartree-Fock Approximation for the Hubbard Model

196 F. V. Andreev, A. V. Kitaev: Connection Formulas for the asymptotics of the fifth Painleve transcendent on the real axis. II

197 J. Dorfmeister, G. Haak: On symmetries of constant mean curvature surfaces

198 Th. Friedrich: On Superminimal Surfaces

199 M. J. Pflaum: The normal symbol on Riemannian manifolds

200 M. U. Schmidt: On complex Bloch-spaces of periodic Schroedinger operators

201 A. Fring, V. Kostrykin, R. Schrader: On the absence of bound-state stabilization through short ultra-intense fields

202 C. Binnenhei: On The Even CAR Algebra

203 I. Kath: $G^{\star} 2(2)-$ Structures on pseudo-Riemannian manifolds

204 W. Müller, M. Schmidt, R. Schrader: Theta functions for infinite period matrices

205 V. M. Adamyan, H. Neidhardt: on the absolutely continuous subspace for contractions and dissipative operators

206 B. D. Dörfel, St. Meißner: Ground state structure and Low Temperature Behaviour of an Integrable chain with Alternating Spins

207 V. M. Adamyan, H. Neidhardt: On the absolutely continuous subspace for non-selfadjoint operators

208 V. Bach, J. Poelchau: Hartree-Fock Gibbs States for the Hubbard Model

209 Ch. Kreft, R. Seiler: Models of the Hofstadter Type

210 M. Lüdde: A Cellular Braid Action and the Yang-Baxter Equation

211 K. Große-Braukmann, K. Polthier: Constant Mean Curvature Surfaces Derived from Delaunay's and Wente's Examples

212 H. Baum, I. Kath: Normally hyperbolic operators, the Huygens property and conformal geometry

213 V. Bach, J. Fröhlich, I. Sigal: Quantum Electrodynamics of Confined Non-Relativistic Particles

214 H. Baum: The Dirac operator on Lorentzian spin manifolds and the Huygens property 
. 